\title{
INDIRECT SEARCH OF DARK MATTER IN SPACE: THEORETICAL AND PHENOMENOLOGICAL ASPECTS
}

\author{
M. CIRELLL \\ CERN Theory Division, CERN, Case C01600, CH-1211 Genève, Switzerland \\ \& IPhT, CNRS, URA 2306 \& CEA/Saclay, F-91191 Gif-sur-Yvette, France \\ * E-mail: marco.cirelli@cea.fr \\ ipht.cea.fr
}

\begin{abstract}
Dark Matter constitutes more that $80 \%$ of the total amount of matter in the Universe, yet almost nothing is known about its nature. A powerful investigation technique is that of searching for the products of anuihilations of Dark Matter particles in the galactic halo, on top of the ordinary cosmic rays. Recent data from the PAMELA and FERMI satellites and a number of balloon experiment have reported unexpected excesses in the measured fuxes of cosmic rays. Are these the first direct evidences for Dark Matter? If yes, which DM models and candidates can explain these anomalies (in tems of annihilations) and what do they imply for future searches? What are the constraints from gamma rays measurements and cosmology? [Saclay T'-09/222, CERN-PH-TH/2009-252]
\end{abstract}

\section{Introduction}

While compelling evidence for the existence of Dark Matter (DM) now comes from a number of astrophysical and cosmological probes, no explicit detection has been confirmed yet. The indirect detection strategy relies on the possibility of seeing signals of the presence of DM in terms of the final products $\left(e^{ \pm}, p, d, \gamma, \nu \ldots\right)$ of DM annihilations in the galactic halo, on top of the ordinary cosmic rays. The recent positive results from a number of indirect detection experiments have suggested the possibility that indeed such a signal has been seen.

- Data from the PAMELA satellite show a steep increase in the energy spectrum of the positron fraction $e^{t} /\left(e^{+t}+e^{-m}\right)$ above $10 \mathrm{GeV}$ ip to $100 \mathrm{GeV}$.

- Data from PAMELA also show no excess in the $\bar{p} / p$ energy spectrum compared with the predicted background.

- The balloon experiments ATIC-2 and PPB-BETS report the presence of a peak in the $e^{+}+e^{-}$energy spectrum at around $500-800 \mathrm{GeV}$.

- This sharp feature is however questioned by results of the FERMI satellite: an excess exists but the $e^{+}+e^{-}$spectrum is found to be a power law.

- The HESS telescope also reports the $e^{+}+e^{-}$energy spectrum above energies of $600 \mathrm{GeV}$, showing a power law spectrum in agreement with the one from FERMI and eventually a steepening at energies of a few TeV.

In this presentation I will address the following issues (the discussion in based on ${ }^{1-4}$ ):

- Which characteristics must a DM candidate have to fit the above data?

- What are the constraints from other observations (e.g. galactic $\gamma$ rays)?

- Are there constraints from cosmological observations? 


\section{2. $e^{+}, e^{-}$and $\bar{p}$ : which Dark Matter can fit the data?}

We perform fits to the data listed above, smoothly scanning over the charged CR propagation configurations and DM halo models, within the boundaries described in. ${ }^{1}$ Moreover, we assume that the $e^{+}, e^{-}, \bar{p}$ background spectra can be freely renormalized, and have independent \pm 0.05 errors in their energy slope. This mimics the main uncertainties in astrophysical backgrounds.

First, we consider the fit to PAMELA $e^{+}$data only (16 data points). We find that DM amnihilations into $e, \mu, \tau, W$ can reasonably well reproduce the data for any DM mass, while amnihilations into $Z, t, q, b, h$ give a good fit for DM heavier than about $1 \mathrm{TeV}$. Next, we add the PAMELA $\ddot{p} / p$ data (17 data points). Annihilation into leptons are not constrained as they do not produce $\ddot{p}$. On the contrary, all other channels are significantly constrained, and allowed only if the DM particle is heavier than almost $10 \mathrm{TeV}$. Only in such a case the $\bar{p}$ excess lays at energies above those explored currently by PAMELA. The bound dominantly comes from high energy data where the solar modulation is negligible. The implications of the complementarity of PAMELA $e^{+}$and $\bar{p}$ data on constraining new physics are therefore evident. We then add to the fit the balloon data (37 points in total). Because they show a sharp cut-off in the excess just below $1 \mathrm{TeV}$, the DM mass should be close to $1 \mathrm{TeV}$, and all other but leptonic DM annihilation channels are strongly disfavored or excluded. More precisely, DM amnihilations into $\mu$ seem to give the best fit. Finally, replacing the balloon data by the FERMI and HESS data modifies slightly the best fit in favor of a candidate with a somewhat higher mass $(\sim 3 \mathrm{TeV})$ and a channel producing somewhat smoother leptonic spectra such as $\tau \tau$.

The last important point concerns the values of the annihilation cross section which are required in order to fit the data (for a given mass and given primary annihilation channel). Values of the order of $10^{-23} \mathrm{~cm}^{3} / \mathrm{sec}$ or more (for the masses under consideration) are needed. These are much larger than the typical cross section required by DM thermal production in cosmology $\left(\sim 3 \cdot 10^{-26} \mathrm{~cm}^{3} / \mathrm{sec}\right)$. They can be justified in specific models in terms of some enhancement mechanism which is effective today but not in the early universe.

\section{Constraints from prompt, ICS gamma rays and radio}

Given these tantalizing but surprising hints of Dark Matter annihilations in the charged particle signals, it is now crucial to consider the constraints on this interpretation that come from the photon fluxes that necessarily accompany them. These photon fluxes are produced:

i) Directly as a product of the DM annihilations themselves (mainly from the bremsstrahlung of charged particles and the fragmentation of hadrons, e.g. $\pi^{0}$, produced in the amihilations), at energies comparable to the DM mass $M$, i.e. in the $\gamma$-ray energy range of tens of $\mathrm{GeV}$ to multi- $\mathrm{TeV}$.

ii) By the Inverse Compton Scattering (ICS) of the energetic $e^{ \pm}$, produced in 
the DM amnihilation, onto the low energy photons of the CMB, the galactic star-light and infrared-light, which are thus upscattered in energy.

iii) At much lower energies, e.g. radio frequency, by the synchrotron radiation emitted in the galactic magnetic field by the $e^{ \pm}$from DM annihilations.

The best targets to search for these annihilation signals are regions with high DM densities, such as the Milky Way Galactic Center (GC), the Milky Way Galactic Ridge (GR) and the Sagittarius Dwarf spheroidal satellite galaxy (Sgr dSph). In the case of the ICS signal, however, an intense flux is expected also from the regions of the galactic halo outside of the messy GC region, a feature which makes this a very interesting and more robust signature. The predicted photon fluxes can then be compared with observational data, in order to rule out combinations of astrophysical and particle physics parameters that violate observational constraints. The results show that the regions identified by charged CR are excluded by these constraints. It is possible to relax them by assuming a less steep DM profile, such as isothermal (less DM concentration in the GC region leads to less gamma ray production), which is however disfavored by numerical simulations. For a full discussion, see Ref. ${ }^{2}$ and ${ }^{3}$.

\section{Constraints from the reionization history}

The flux of energy injected by DM annihilation (all the way from the recombination epoch down to today through the formation history of DM halos) results in ionization (and heating) of the intergalactic medium. One way to constrain DM annihilation properties is therefore to look at the total optical depth of the Universe $\tau$, which results from the free electrons produced by the ionization. $\tau$ is measured by WMAP to be $\tau=0.084 \pm 0.016$, of which about 0.038 due to the low-redshift reionization $(z<6)$ produced by stars. A DM-induced optical depth larger than 0.062 (the $1 \sigma$ upper bound of the above) is therefore excluded by these arguments. Again one finds that large portions of the regions that allow to fit the PAMELA and FERMI+HESS CR excesses in term of DM annihilations are ruled out by the optical depth bound. E.g. the entire PAMELA and FERMI+HESS region for the $\tau^{+} \tau^{-}$or $\mu^{+} \mu^{-m}$ case around $m_{\chi}=2$ or $3 \mathrm{TeV}$ is excluded. Full discussion in Ref. ${ }^{4}$.

\section{Acknowledgments}

I thank my collaborators A.Strumia, G.Bertone, M.Kadastik, F.Iocco, P.Panci, M.Raidal and M.Taoso. I thank the EU ITN "UniverseNet" (MRTN-CT-2006035863). No need to thank the organizers of the MG12 for an enjoyable conference.

\section{References}

1. M. Cirelli, M. Kadastik, M. Raidal and A. Strumia, Nucl. Phys. B 813 (2009) 1.

2. G. Bertone, M. Cirelli, A. Strumia and M. Thoso, JCAP 0903 (2009) 009.

3. M. Cirelli and P. Panci, Nucl. Phys. B 821 (2009) 399.

4. M. Cirelli, F. Iocco and P. Panci, JCAP 0910 (2009) 009. 
